\title{
Dynamic Activity-Related Incentives for Physical Activity
}

\author{
Julia Schüler, Sibylle Brunner \\ University of Zurich, Zurich, Switzerland \\ Email: j.schueler@psychologie.uzh.ch
}

Received December $7^{\text {th }}$, 2011; revised January $15^{\text {th }}$, 2012; accepted January $21^{\text {st }}, 2012$

\begin{abstract}
The present studies adopted the theoretical framework of activity- and purpose-related incentives (Rheinberg, 2008) to explain the maintenance of physical activity. We hypothesized that activity-related incentives (e.g., "fun") increase more than purpose-related incentives (e.g., "health") between the initiation and maintenance phase of physical activity. Additionally, change in activity-related incentives was hypothesized to be a better predictor of maintenance of physical activity than change in purpose-related incentives. Two correlative field studies with rehabilitation patients (Study 1) and Nordic Walkers (Study 2) were conducted to test the hypotheses. Participants' incentives of physical activity were measured at the beginning of exercising and two weeks (Study 1; T2) and three months (Study 2; T2) later. At T2, participants were asked for their current physical activity. Both studies showed a greater change of activity-related incentives than purpose-related incentives. Furthermore, change in activity-related incentives was more predictive of the maintenance of physical activity than change in purpose-related incentives. The results showed the important role of activity-related incentives in maintenance of physical activity. The theoretical contribution to physical activity maintenance research and practical implications for health promotion programs were discussed.
\end{abstract}

Keywords: Physical Activity; Behavior Maintenance; Motivation; Incentives

\section{Introduction}

While people are generally aware of the favorable health benefits associated with regular physical activity, exercise and sport (Blair \& Connelly, 1996), many people fail to persist in being physically active and performing sport and exercise behavior. A high percentage of individuals who initiate physical activity fail to maintain this behavior and drop-out within the first six months (50\% according to Wing, 2000: p. 85). Thus, researchers are interested in the factors that contribute to the adherence of physical activity (see Dishman, 1994; Markland \& Hardy, 1993) and suggest different determinants such as goals (e.g., Duda, 1989), commitment to physical activity (Scanlan, Carpenter, Schmidt, Simons, \& Keeler, 1993), self-efficacy beliefs (Schwarzer, 1992) and perceived satisfaction with received outcomes of physical activity (Rothman, 2000).

In the present paper, we suggest that incentives might be partly responsible for the maintenance of physical activity. In contrast to previous approaches on related concepts such as personal exercise incentives (e.g., Duda \& Tappe, 1989) and exercise motivation (Markland \& Hardy, 1993), our approach is strongly based on the classical concept of incentives in motivational psychology ${ }^{1}$ (e.g., Atkinson, 1957; Beckmann \& Heckhausen, 2008; Vroom, 1964) and on an extension of the classical concept (Rheinberg, 2008). Here, incentives are defined as stimuli that refer to affective goal-states and thereby stimulate goal-directed behavior (Beckmann \& Heckhausen, 2008). At first sight the incentive concept seems to be related to research on outcome expectancies. Most expectancy-based theories draw on the idea that "people's actions are greatly affected by their

\footnotetext{
${ }^{1}$ Note that the term "incentive" is not used here in the sense of an external reward (as is the case in research on the undermining of intrinsic motivation; e.g., Deci, 1971), but is defined as a situational characteristic that promises the satisfaction of a need.
}

beliefs about the probable outcomes of those actions" (Scheier \& Carver, 1987: p. 170; for the domain of physical activity see Anderson, Wojcik, Winett, \& Williams, 2006). They therefore understand expectancies as anticipations of desired or undesired future states. These outcome expectancies lead, for example, to an optimistic orientation that helps people to strive effectively for their goals (Scheier \& Carver, 1987). Although the outcome expectancy concept shares some characteristics with incentives, for example the fact that they both have motivating functions, they are quite different in their defining features. In terms of classical expectancy-value models in motivation psychology (Atkinson, 1957), outcome expectancies represent the general belief that it is worth striving for a goal and initiating goal-directed actions. They are cognitive evaluations of the probability of goal-attainment (e.g. it is highly probable); however, nothing is said about the valence and the content of the expected outcome. This is addressed by the value component of the expectancy-value model, which defines the future state (fun, health) and defines whether it is desirable (health) or undesirable (illness). In summary, theoretically expectancies and incentives are both important reasons for individuals to behave in a certain kind of way (e.g., performing sports regularly), but are conceptually different. Two further reasons for relying on the concept of incentives rather than on outcome expectancies are that empirical investigation of the latter yield mixed results regarding how strong and for whom outcome expectancies predict physical activity (e.g., Williams, Anderson, \& Winett, 2005 , for a review) and that the concept of incentives allows activity- and purpose-related incentives to be distinguished, which is the most important aspect of our research question.

In accordance with Rothmans' (2000: p. 64) statement that "the psychological factors that enable people to adopt a new pattern of behavior are not necessarily those that help them to sustain that behavior over time", we assume that incentives that 
stimulate the initiation of physical activity differ from those that are responsible for its maintenance. In other words, incentives for being physically active are dynamic rather than static. To empirically test this assumption, we refer to the concept of activity- and purpose-related incentives proposed by Rheinberg (2008). When asked for their incentives of physical activity, individuals often mention "fun”, “enjoyment”, "social affiliation”, "health”, "improving appearance” or "loosing body weight” (e.g., Markland \& Ingledew, 2007). According to the definition of incentives, all examples represent positive, affective goal-states that stimulate a person to engage in physical activity. Besides this common characteristic, however, for example "fun" versus "health" obviously differ and it is these differences upon which we focus in analyzing their role in the initiation and maintenance of physical activeity. Since one striking difference between "fun" and "health" lies in the fact that the former is related to the physical activity itself and the latter to the outcome of being physically active, we adopt the classification suggested by Rheinberg (2008) according to which incentives are classified as activity-related and purposerelated.

Following a more detailed introduction to the concepts of activity- and purpose-related incentives, we will deduce two hypotheses which assume that the activity-related and purpose-related incentives differentially increase over time between initiation and maintenance phase of physical activity and that these increases have varying power in the prediction of long-term maintenance of physical activity.

\section{Activity-Related and Purpose-Related Incentives}

Incentive approaches in motivation psychology take into account that individuals are not only passively driven by needs and motives but are also able to anticipate positive future states (e.g., Atkinson, 1957; Beckmann \& Heckhausen, 2008) and direct their behavior towards these states. Traditional research on motivation postulates that anticipated future states lie in the result of an action (Atkinson, 1957; McClelland, 1985; Vroom, 1964): An action such as regularly exercising is attractive and meaningful if the consequences of the action, for instance improved health, are attractive. This also applies to the Extended Cognitive Model (Heckhausen \& Rheinberg, 1980) in which incentives are located at the end of an action course. Since this model is not able to explain behavior that is performed without or with less pronounced purpose-related incentives, such as being physically active without aiming to improve health, or riding a bicycle without a destination, Rheinberg (2008) extended the original model by acknowledging that while purpose-related incentives are important, they are not the only type of incentives for human behavior. He assumed a second source of incentives which lies in the action itself; so-called activity-related incentives. With respect to physical activity, people may exercise because it is fun, because they enjoy the movements and the feeling of muscular power, or because of other positive experiences. We assume that a detailed analysis of activity- and purpose-related incentives for physical activity, respectively, will help to shed light on the phenomena of behavior initiation and maintenance.

The terms activity- and purpose-related incentives are conceptually similar to the terms intrinsic and extrinsic motivation (e.g., Deci \& Ryan, 1985, 2000). One reason for nevertheless choosing the terms "activity-" and "purpose-related incentives" is that the terms intrinsic and extrinsic are used with different foci by different authors within, for example, self-determination theory (SDT) research (Deci \& Ryan, 2000), interest research (Sansone \& Smith, 2000), and motivation research (Heck- hausen \& Heckhausen, 2008; Shah \& Kruglanski, 2000) (for a summary of different meanings of "intrinsic" see Rheinberg, 2008). The most important reason for using the terms "activity-" and "purpose-related incentive" is that these terms most clearly focus on the very feature which we assume to be the key difference between incentives such as "fun" and "health" and thus best explain the differential effects of activity- and purpose-related incentives on maintenance of physical activity. This difference comprises the temporal distance from the activity itself. For example, the activity-related incentive "fun" is very closely temporally related to the activity and is experienced directly during the course of action itself. In contrast, purpose-related incentives such as "health" are by definition separated from the activity, constituting a consequence which is experienced when the activity has been terminated. We assume that this difference regarding the temporal distance from the activity is accompanied by two effects that will be explained in the following section.

\section{Differential Changes in Activity- and Purpose-Related Incentives}

We assume that incentives are not "stable characteristics of person-environment interactions” (McClelland, 1985: pp. 180181) but are rather subject to dynamic change from the initiation to the maintenance phase of behavior. And we expect activity- and purpose-related incentives to differentially change over time. A change in activity-related incentives is plausible due to the very nature of such incentives: Most activity-related incentives (e.g., having fun, feeling good whilst performing a perfect movement) require affective and somatic experience of the activity itself and can therefore only develop when the activity is repeatedly performed over a certain length of time. They often involve body sensations or psychological sensations and are thus hard to anticipate upon first beginning to exercise. This theorizing was supported in the sport domain by McAuley, Wraith and Duncan (1991) who pointed out that external reasons such as improvement of physical appearance might be the reason for adopting exercise behavior and physical activity, but that intrinsic reasons might become more salient across the time, because people's skills and physical conditioning improve and enable more enjoyment of physical activity. These considerations are further supported by current research on intrinsic motivation, interest, and flow-experience which suggesting that some incentives are inherent in the activity itself and are likely to be discovered in long-term interaction with the activity (Deci \& Ryan, 1985; Csikszentmihalyi, 1990; "expertise effect of flow” by Rheinberg, 2008).

While due to our line of argumentation activity-related incentives existentially depend upon the activity, purpose-related incentives require less experience of the activity itself, given that they are more explicit from the very onset and are therefore more easily anticipated. It is, for example, common knowledge that exercising and physical activity improves health and regulates body weight. We hypothesized that purpose-related incentives would increase less from the initiation to the maintenance phase than activity-related incentives. Based on this sum of theoretical and empirical evidence, we deduce our first hypothesis that incentives are dynamic and change from the initia- 
tion to the maintenance phase of an activity, with activity-related incentives such as "fun" increasing more than purposerelated incentives such as "health".

The idea that incentives change across the time was proposed in a broader sense by organismic integration theory which is an SDT subtheory (Deci \& Ryan, 1985). It postulates that much behavior is not intrinsically, but extrinsically motivated. This is especially true after early childhood when social pressure and demands require doing activities that are not interesting (Ryan \& Deci, 2000). Therefore, during childhood socialization as well as across the life span, processes of internalization and integration of extrinsic demands into the self enable a more self-regulated (rather than externally regulated) behavior. For example, individuals take in external regulation (introjection), identify with a behavior's value (identification) or they integrate external regulations with the aspects of their self (integration). However, also the highest transformation of externallyregulated into self-regulated behavior is a form of extrinsic motivation and thus still differs from intrinsic motivation (in terms of doing the activity for its own sake).

Other concepts related to our idea of the dynamicity of incentives stems from researchers examining changes in the salience of different sorts of incentives across time. Although these researchers differed in the labeling and conceptualization of "incentive", the phenomena they investigated are rather similar. Liberman and Trope (1998; Trope \& Liberman, 2003), for example, analyzed temporal changes in abstract incentives ("highlevel construals") and concrete incentives ("low-level construals”) and Pennington and Roese (2003) found that promotion incentives ("promotion goal importance") increased with temporal distance whereas prevention incentives ("prevention goal importance") remained stable over time.

While previous research focused on the change of behavior regulation through internalization or integration or on weighting of and interplay between positive and negative incentives (Prochaska et al., 1994) or on the salience of incentives across time (Liberman \& Trope, 1998) as reported above, the present paper divided incentives into the two categories activity- and purpose-related incentives, observed their variation across time, and analyzed their effects on maintenance of physical activity. To summarize, the present paper adheres to the notion that incentives are not stable but differ across time and further assumes that the differentiation between activity- and purposerelated incentives is an important concept that enhances the predictability of behavior maintenance.

\section{The Differential Predictive Power of Activity- and Purpose-Related Incentives}

The second hypothesis derived from theoretical considerations on activity- and purpose-related incentives concerns the effects of incentive changes on the maintenance of physical activity. We assume that physical activity is more likely to be maintained by those individuals for whom activity-related incentives increase during physical activity, than by those participants for whom purpose-related incentives increase. The rationale behind this assumption is based upon the difference in temporal distances from the activity. The positive quality of activity-related incentives, such as having fun or enjoying movements, is very closely temporally related to the activity itself and thus functions as a direct reward for activity performance. According to the principle of operant conditioning
(Skinner, 1938), this should enhance the probability of the activity being repeated. In contrast, individuals whose activityrelated incentives do not increase from the initiation to the maintenance phase miss out on an important source of motivation and are subsequently more endangered when it comes to disengaging from the behavior. Unlike activity-related incentives, purpose-related incentives, such as "health" or losing body-weight, are often long-term effects that can seldom be experienced immediately following physical activity. Due to the less intense temporal relationship between purpose-related incentives and the activity, the rewarding effect of these incentives should thus be much weaker than that of activity-related incentives (for the effects of time-association between action and reward see Tarpy \& Sawabini, 1974; Shanks \& Dickinson, 1991). To summarize, we postulate that an increase in activity-related incentives predict the maintenance of physical activity better than an increase in purpose-related incentives.

\section{The Present Research}

We postulate greater increases in the activity-related incentives, as for example "fun" than in the purpose-related incentives, as for example "health" from the initiation phase to the maintenance phase of physical activity (Hypothesis 1). Furthermore, increases in activity-related incentives are hypothesized to be a better predictor of maintenance of physical activity than increases in purpose-related incentives (Hypothesis 2).

Two studies tested these hypotheses. The studies were correlational field studies with performers of fitness sports whose physical activity incentives were measured as predictors of physical activity. Besides these common characteristics, both studies theoretically and methodologically complement one another in three important ways. First, Study 1 focused on the incentives of rehabilitation patients participating in physical activity courses provided by a rehabilitation centre and Study 2 measured incentives among middle-aged Nordic walkers. With using different study samples we aimed to support the generalizability of the study results. The second difference between the two studies was the time interval of data collection. In Study 2, data were collected on two occasions, registering incentives two weeks apart. Study 2 extended this time period by measuring incentives with a three-month interval and predicted the maintenance or cessation of physical activity six months after its initiation. The third but very important aspect distinguishing the studies is how we assessed the incentives. In Study 1 the incentive "fun" as a prototype of an activity-related incentive and "health" as a purpose-related incentive prototype were presented and participants rated how much the incentives fit to their person. Study 2 aimed at extending the incentive measure by adding incentives from the achievement, affiliation and power domain that were gained by an expert rating.

\section{Study 1}

In Study 1 incentives for health-related physical activity were measured using a questionnaire administered on two separate occasions; in the last week of a health-oriented physical activity course that was integrated in a rehabilitation program and two weeks later after participants had left the rehabilitation center. Participants were thus accompanied from their initiation of physical activity up to the time when they either maintained or disengaged from exercising. 
Due to practical reasons we had to use a brief measure of incentives and thus choose the incentives "fun" and "health" as representative activity- and purpose-related incentives for the initiation and maintenance of physical activity. We investigated whether incentive changes predicted actual physical activity by asking participants whether they had maintained their recommended frequency of physical activity over a set period of time ("optimal exercisers") or had exercised less than recommended ("suboptimal exercisers"). This criterion of physical activity was selected due to our assumption that no patient would completely quit the recommended physical activity program due to a high level of internal pressure (physical activity is needed to reduce pain) and a high level of external pressure (recommendation of physical activity by a person of authority; external control of compliance after two weeks) but that patients would vary in the amount of physical activity performed. According to our first hypothesis, the activity-related incentive "fun" should increase more compared to the purpose-related incentive "health" within the two-week period for the maintainers but not for suboptimal performers. According to our second hypothesis, the change in "fun" should be a better predictor for optimal exercising versus suboptimal exercising than the change in "health".

\section{Method}

\section{Participants and Procedure}

Forty-seven male and 20 female participants of a health physical activity program in a rehabilitation clinic were contacted and asked to participate in a study on "motivation and experiences of physical activity". Patients with different diagnoses including arthrosis, low back pain, and fibromyalgia, aged between 24 and 79 years $(M=55.04, S D=11.97)$, who participated in a threeweek health program in the clinic took part in the present study. For each participant, physiotherapists compiled an individual physical activity program consisting of various training elements such as aqua-fit, cycling, or swimming. The physiotherapists highly recommended continuing this physical activity program after being discharged from the rehabilitation clinic. Participants were recruited during the final week of the program by a female experimenter who explained the data collection procedure in detail and who directly answered any ensuing questions. The study consisted of two parts. First, participants completed a questionnaire at the beginning of the final physical activity lesson (T1) and returned it to the experimenter. The questionnaire comprised baseline measures of the incentives "fun" and "health" and also asked for details on the specific physical activity program that had been individually arranged for each participant. Two weeks after the end of the health physical activity program (T2), participants were contacted via mail and asked to respond to questions concerning their current incentives and physical activity.

\section{Incentive Assessment}

The two incentives "fun" and "health" were assessed using the two statements "My reason for being physically active is fun" and "My reason for being physically active is health". Participants rated the degree to which the statements personally applied to them (1: not at all - 5: very much). Incentive changes were assessed by using difference scores that is by subtracting T1 ratings from T2 ratings separately for "fun" and "health".

\section{Assessment of Physical Activity}

In order to evaluate whether a participant had continued to perform the recommended physical activity program after having been discharged from the clinic, we compared the physical activity reported at T2 with the recommendations made by the physiotherapists. Recommendations were assessed by asking participants at T1 about the customized physical activity program which had personally been recommended to them for continued performance by the physiotherapist. Two weeks after finishing the clinic physical activity program (T2), participants were asked about their current physical activity. Based on this information, participants were subsequently divided into two groups: optimal exercisers $(N=42)$ who reported performing the recommended physical activity program and suboptimal exercisers $(N=25)$ who reported performing less than the recommended physical activity program. As expected, none of the patients reported having completely quit the physical activity program.

\section{Results}

\section{Preliminary Analyses}

Preliminary analyses revealed no significant impact of age, clinical diagnosis, or recommended physical activity. Men and women did not differ in any of the relevant variables and sex did not influence the results reported below. So data were collapsed for the following analyses.

\section{Descriptive Statistics and Intercorrelations between Variables}

"Fun" and "health" incentive-changes scores were not significantly associated (Pearson correlation, $r=.20$, n.s.). Changes in the "fun" incentive $(M=-.02, S D=.79)$ were significantly correlated with the dichotomous variable optimal exercise versus suboptimal exercise (Spearman's rho, $r=.31, p<.05$ ), indicating that the higher the positive change of "fun", the more likely the participant was to continue their personally recommended physical activity program. Change in the "health" incentive $(M=-.05, S D=.60)$ was not significantly associated with optimal physical activity versus suboptimal physical activity (Spearman's rho, $r=-.08$, n.s.).

\section{Incentive Change in Optimal and Suboptimal Exercisers}

Given our assumption that incentives would differentially change for optimal exercisers and suboptimal exercisers, the incentive changes are illustrated separately for each of these two groups in Figures 1(a) (optimal exercisers) and 1(b) (suboptimal exercisers). The optimal exercisers are characterized by an increase in "fun" (T1: $M=4.31, S D=.78$; T2: $M=4.50, S D$ $=.59$ ) and a relatively stable "health" incentive (T1: $M=4.62$, $S D=.58$; T2: $M=4.55, S D=.63$ ). Statistical significance of the difference between the changes in "fun" and "health" incentives was tested by comparing the incentive- change indexes (difference score) in a repeated-measure analysis of variance. The incentive-change index for "fun" ( $M=.19, S D=.09)$ was significantly higher than the index for "health" $(M=-.07, S D$ $=.09), F(1,41)=4.52, p<.05$.

Figure 1(b) shows that suboptimal exercisers are characterized by a decrease in the activity-related incentive "fun" (T1: $M=$ 4.28, $S D=.94$; T2: $M=3.93, S D=1.25$ ) and a relatively stable purpose-related incentive "health" (T1: $M=4.68, S D=.69$; T2: $M=4.72, S D=.46)$. The repeated-measure analysis of variance showed significantly greater decreases in the "fun" incentivechange index $(M=-.35, S D=.19)$ than the "health" incentive-change index $(M=.05, S D=.12), F(1,24)=5.21, p<.05$. 


\section{1a) Incentive change in optimal exercisers}

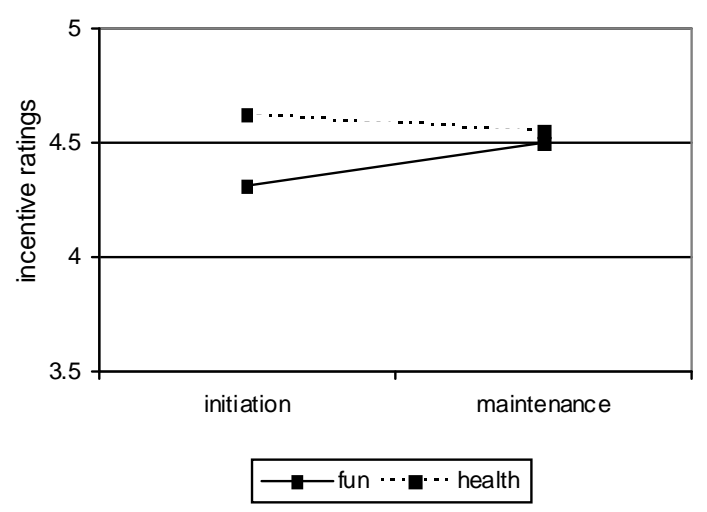

(a)

1b) Incentive change in suboptimal exercisers

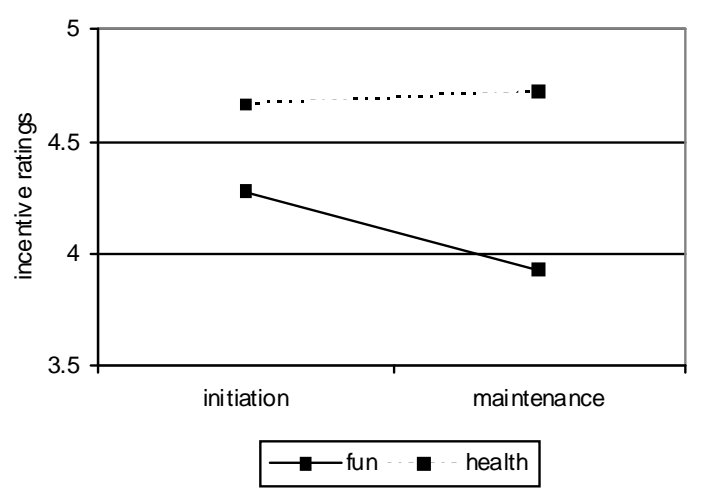

(b)

Figure 1.

Change in the incentives "fun" and "health" from the initiation (T1) to the maintenance (T2) phase of physical activity for optimal exercisers $(N=42)$, see Figure 1(a) and suboptimal exercisers $(N=25)$, see Figure 1(b).

\section{Effects of Incentive Change on the Maintenance of Physical Activity}

A step-wise binary logistic regression analysis was conducted to test whether change in the "fun" incentive or the "health" incentive was a better predictor of becoming an optimal versus suboptimal exerciser. The two types of incentive changes (difference scores) were entered into the regression equation in a forward step-wise manner in order to investigate the influence of each variable on the dependent variable. The constant was entered first followed by the independent variable which correlated most highly with the dependent variable and then the final variable. In this way, variables with low predictive power were identified and subsequently eliminated from the regression equation (criterion: Wald statistic). Step-wise binary linear regression analysis thus indicated which of the two incentive changes best predicted whether a participant showed optimal or suboptimal physical activity and which incentive change might be excluded from the regression equation because it did not account for additional variance. It was shown that change in the "fun" incentive should be included as predictor in the regression model (Wald (1) =6.67, $p<.05 ; B=1.0, S_{e}=.39$ ), whereas change in the "health" incentive did not explain any additional variance in physical activity and was subsequently excluded. The chi-square test of model fit (chi-square $=7.95, p<.01$ ) indicated a significantly better goodness of fit for the model which included change in the "health" incentive (Model 2) than the model which contained only the constant (Model 1). Model 2 explained $15.5 \%$ of variance in the dependent measure (Nagelkerke's $R$-square $=.155$ ) that is optimal versus suboptimal exercising.

\section{Brief Discussion Study 2}

Study 1 confirmed our hypotheses in a sample of participants of a rehabilitation physical activity program. Participants who continued to perform their recommended physical activity program two weeks after discharge (optimal exerciser) showed a different pattern of incentive change than those participants who exercised less than recommended (suboptimal exercisers). In accordance with our hypothesis, optimal exercisers were characterized by a greater positive change in the "fun" incentive than the "health" incentive. Suboptimal exercisers were characterized by a decrease in the "fun" incentive and a relatively stable level of the "health" incentive. As postulated in our second hypothesis, change in the "fun" incentive also proved to be a significant predictor of optimal versus suboptimal physical activity, whereas change in the "health" incentive lacked predictive power. These results confirmed the stronger effect of the activity-related incentive "fun" on the maintenance of physical activity. However, one might criticize that the two-week period is too short to measure changes in incentives and behavior adequately. Another limitation is the single item measure of activity- and purpose-related incentives. Study 2 addressed these issues.

\section{Study 2}

Study 2 used a longitudinal design to examine whether incentive change predicts the long-term maintenance of physical activity. According to the Transtheoretical Model (Prochaska \& DiClemente, 1983, 1992), the different phases through which individuals progress when they change their health-related behavior (see above) can be defined by time criteria. The maintenance phase is assumed to begin when an individual has maintained the behavior change for a period of six months. To fulfill this time criterion, Study 2 accompanied Nordic-walker beginners in their walking activity from their very first lesson in Nordic walking (beginning of the action phase) up to the six-month marker of the maintenance phase.

One aim of Study 2 was to replicate the results found in Study 1 . We expected that the "fun" incentive would increase more than the "health" incentive between the initiation and the maintenance phase and expected that the change in the "fun" incentive would be a better predictor of maintenance than the change in the "health" incentive.

Furthermore, we extended the activity- and purpose-related incentive measure by referring to other important domains of human life rather than fun and health. In order to systemize and reduce the variety of incentives, we refer to dispositional theories on motivation (e.g., McClelland, 1985) which focus on the achievement, power and the affiliation domain. We created an incentive questionnaire in Study 2 that comprises activity- and purpose-related achievement, power, affiliation incentives and some further incentives. Again, we hypothesized that activity-related incentives increased more during the course on being physically active and better predict maintenance of physical activity than purpose-related incentives. 


\section{Method}

\section{Participants and Procedure}

Sixty-eight participants with a mean age of 44.41 years ( $S D$ $=8.60$, range from 29 - 65 years) were recruited from local Nordic walking courses to take part in a study on "first experiences in Nordic walking”. Most participants were female $(N=$ $60)$. The Nordic walking courses were affiliated with a local Nordic Walking Association that offered courses explicitly to beginners with no prior knowledge of Nordic walking. The Nordic walking fitness instructors confirmed that all participants had been correctly classified as beginners.

Participants were recruited in their very first lesson by a female experimenter who explained that data would be collected in three phases in order to obtain a realistic rating of experiences in the first Nordic Walking course. Participants who agreed to take part in the study received a first take-home booklet to be immediately completed upon arriving at home after the first physical activity lesson (T1) and returned via mail. Of 72 beginners who indicated an interest in participating in the study, 68 returned the questionnaire within one week. The questionnaire included baseline measures of incentives and informed the participants that the next phase of data collection would be at the end of the course. At the end of the three-month Nordic walking course (12 weekly lessons; T2), participants once again completed a questionnaire on their incentives for participating in Nordic walking. Fitness instructors additionally informed the participants about upcoming opportunities for participating in Nordic walking (e.g., Nordic walking routes; further courses) and that they would be contacted three months later by the experimenter with a further questionnaire on their Nordic walking activities. Six months after beginning the course, participants were contacted via mail and were questioned with respect to their current Nordic walking activities (T3). After returning the final questionnaire, participants were debriefed about the research question and hypotheses.

\section{Incentive Assessment}

"Fun" and "health" incentives were assessed using ratings of the statements "I do Nordic walking because it is fun/it improves my health” (1: not at all - 5: very much). Again, incentive change was measured by subtracting ratings at $\mathrm{T} 1$ from ratings at $\mathrm{T} 2$.

The items of the extended incentive measure were the result of a two-step procedure. First, two experts on motivation research who are highly familiar with the concept of activity- and purpose-related incentives, and the theoretical concept of achievement, power and affiliation motives generated activity- and purpose-related incentives for the achievement, power and affiliation domain independently from each other. Secondly, the two best fitting items of each category were chosen by the experts discussing the items in detail. The activity-related incentive scale comprises two incentives for the achievement, affiliation and power domain (e.g., achievement: I do Nordic Walking/because I like to experience progress; affiliation: because I enjoy spending time with my friends; power: because I experience a sense of power) and a further item that we expected to be important but did not fit in one of the categories (because I get absorbed by the activity). Participants rated their agreement to each of the seven statements using a 5-point scale from 1 (not at all) to 5 (very much). The scale was sufficiently reliable (T1: $\alpha=.67$; T2: $\alpha$ $=.71$ ). The mean across all items was computed (T1: $M=3.31$,
$S D=.63 ; \mathrm{T} 2: M=2.90, S D=.54)$. The purpose-related incentive scale also comprises two items for each of the categories. An example for a purpose-related achievement incentive is "I do Nordic walking because I want to be better than others". An example for purpose-related affiliation incentives is "I do Nordic walking because we often have a night out after the physical activity lessons" and an example for purpose-related incentives in the power domain is "I do Nordic walking because I want to impress others”. Again, a further incentive that people reported when being asked for their reasons of physical activity was added ("I do Nordic walking because I want to lose body-weight"). A purpose-related incentive score was computed by calculating the mean (T1: $M=3.22, S D=.68, \alpha=.62 ; \mathrm{T} 2: M=2.83, S D=.62, \alpha$ $=.71$ ). Activity- and purpose-related incentive change indexes were calculated by subtracting ratings at $\mathrm{T} 1$ from ratings at $\mathrm{T} 2$ (difference scores as in the previous studies).

\section{Assessment of Drop-Out versus Maintenance}

Nordic walking maintenance and drop-out were measured by simply asking participants whether they still performed Nordic walking sports or whether they had dropped out six months after having commenced the course (T3). Forty-nine of the 68 participants $(72.1 \%)$ indicated that they still regularly performed Nordic walking (at least once a week) and 19 participants indicated that they had quit Nordic walking sports.

\section{Results}

\section{Preliminary Analyses and Descriptive Statistics}

Women and men did not differ with respect to any of the study variables. Participants' age also had no significant impact on the results, so that data were pooled for the following analyses. Changes in activity-related incentives $(M=-.09, S D=.58)$ and purpose-related incentives $(M=-.07, S D=.55)$ were significantly correlated (Pearson's $r=.67, p<.001$ ) and activityas well as purpose-related incentives were associated with the maintenance versus drop-out variable (activity-related incentive: Spearman's rho $=-.38, p<.01$, for purpose-related incentive: Spearman's rho $=-.25, p<.05$ ), indicating that the higher participants' incentive changes were, the less likely they would drop out of Nordic Walking sports.

A similar correlation pattern was found for "fun" and "health". Changes in the incentives "fun" $(M=-.15, S D=.65)$ and "health" $(M=-.23, S D=.69)$ were significantly associated (Pearson's $r=.42, p<.01$ ). Both incentives were significantly related to the maintenance versus drop-out variable (Spearman's rho for fun $=-.59, p<.01$, for health $=-.36, p<.01$ ).

\section{Incentive Changes in Maintainers and Drop-Outs}

Given our assumption that incentives would differentially change for maintainers and drop-outs, Table 1 illustrates descriptive statistics for activity- and purpose-related incentives at $\mathrm{T} 1$ and T2 and change indexes separately for each of the two groups. The changes in "fun" and "health" in maintainers and drop-outs were very similar to the change in activity- and purpose-related incentives, except that they were higher. Maintainers reported more fun (T1: $M=4.76, S D=.56$; T2: $M=$ 4.34, $S D=.37)$ as well as health incentives (T1: $M=4.90, S D$ $=.31$; T2: $M=4.82, S D=.44$ ) than drop-outs (activity: T1: $M$ $=4.68, S D=.48$; $\mathrm{T} 2: M=3.95, S D=.85$; purpose: $\mathrm{T} 1: M=$ 4.79, $S D=.41$; $\mathrm{T} 2: M=4.16, S D=.96$ ). For maintainers the fun incentive increased, but decreased for drop-outs. 
Table 1.

Descriptive Statistics for activity- and purpose-related incentives at T1 and T2 and incentive-change indexes for participants who maintained Nordic walking $(N=49)$ and those who dropped out $(N=19)$ (Study 2).

\begin{tabular}{ccccccc}
\hline & \multicolumn{2}{c}{$\mathrm{T} 1$} & \multicolumn{3}{c}{$\mathrm{T} 2$} & \multicolumn{3}{c}{ Incentive change } \\
\cline { 2 - 7 } & $M$ & $S D$ & $M$ & $S D$ & $M$ & $S D$ \\
\hline Maintainers & & & & & & \\
Activity-related incentive & 3.35 & .56 & 3.40 & .62 & .05 & .43 \\
Purpose-related incentive & 2.95 & .56 & 2.96 & .62 & .00 & .47 \\
Drop-outs & & & & & & \\
Activity-related incentive & 3.21 & .81 & 2.67 & .61 & -.48 & .56 \\
Purpose-related incentive & 2.77 & .48 & 2.47 & .50 & -.34 & .52 \\
\hline
\end{tabular}

On a descriptive level, the group of maintainers showed a higher incentive-change index for activity-related incentives $(M$ $=.05, S D=.51$ ) compared to the incentive-change index for purpose-related incentives $(M=.01, S D=.53)$. However, a repeated-measure analysis of variance revealed that the differences were not significant, $F(1,48)=.20$. In contrast, for drop-outs the activity-related incentives $(M=-.44, S D=.61)$ decreased significantly more than the purpose-related incentives $(M=-.30$, $S D=.53), F(1,18)=9.91, p<.01$.

The "fun" and "health" incentive change for maintainers and drop-outs was comparable to the incentive change of optimal and suboptimal exercisers in Study 1 (e.g., illustrated in Figure 1). The incentive change for "fun" $(M=.08, S D=.45)$ was marginally higher than the incentive-change index for "health" $(M=$ -.08, $S D=.53), F(1,48)=3.35, p=.07$. Drop-outs were mainly characterized by a decrease in "fun" $(M=-.74, S D=.73)$ and "health" $(M=-.63, S D=.90)$ incentives that did not significantly differ $(F(1,18)=.20, p=.63)$.

\section{Effects of Incentive Change on the Maintenance of Physical Activity versus Drop-Out}

Similar to Study 1, a step-wise binary logistic regression analysis was used to examine whether activity- and purposerelated incentive changes predicted Nordic-walking maintenance or drop-out six months after initiation of the physical activity. The activity-related incentive change predicted exercise in Nordic-walking maintenance and was thus included in the regression model (Wald $(1)=8.55, p<.01 ; B=-1.61, S_{e}$ $=.55)$. In contrast, change in purpose-related incentives failed to reach significance and was thus excluded due to a lack of predictive power. The chi-square test of model fit $(10.17, p$ $<.01$ ) indicated a highly significant difference in the goodness of fit for the model which included change in activity-related incentives (Model 2) compared to the model which contained only the constant (Model 1) (Nagelkerke's $R$-square $=.20$ ).

Similar to the activity- and purpose-related incentive measure, the change in the "fun" incentive was found to predict physical activity maintenance and was included in the regression model (Wald $(1)=13.62, p<.001 ; B=-3.12, S_{e}=.84$ ). The change in the "health" incentive was thus excluded from the regression equation. The chi-square test of model fit (26.45, $p<.001$ ) indicated a highly significant difference in the goodness of fit for the model which included change in the "fun" incentive (Nagelkerke's $R$-square $=.46$ ).

\section{Brief Discussion Study 1}

The present study accompanied Nordic walkers from the ini- tiation phase up to the maintenance phase (six months later) of physical activity. In line with Study 1, results revealed different patterns of incentive change for those participants who maintained Nordic walking behavior and those who dropped out after six months. For maintainers, the "fun" incentive increased stronger than the "health" incentive. Conversely, for drop-outs, "fun" decreased more (but not significantly) than "health". On a descriptive level maintainers showed a greater increase in activity-related incentives than in purpose-related incentives. Conversely, drop-outs were characterized by a stronger decrease in activity- compared to purpose-related incentives. A binary logistic regression analysis again confirmed our second hypothesis: change in activity-related incentive change was included in the regression equation as a significant predictor of maintenance versus drop-out, whereas change in purpose-related incentives was excluded due to a lack of predictive power.

\section{General Discussion}

Based on considerations that the experience of physical activity might change individuals' incentives (see also Rothman, 2000), we empirically demonstrated and thus conclude that incentives of physical activity are not static but rather change over time. This idea of dynamicity corresponds to the postulate of modern health theories of behavior change, that the course of complex human action consists of qualitatively different action phases in which different variables-in our case incentivesare active (Prochaska \& DiClemente, 1983; Rothman, 2000; Schwarzer, 1992). As an extension of previous theoretical considerations, we postulated that not only the dynamic change in incentives but also the differentiation between two types of incentives must be considered when it comes to predicting behavior maintenance. According to Rheinberg's (2008) differentiation between activity- and purpose-related incentives, stimuli which incite goal-directed behavior (in our case physical activity) are either related to the physical activity itself (e.g., fun) or to the result of the activity (e.g., health). We assumed that these two types of incentives differentially change over time and differentially influence physical activity. Since activity-related incentives are dependent on the experience of the activity and are discovered in interaction with it (Csikszentmihalyi \& Rathunde, 1992), they were expected to increase more strongly than purpose-related incentives, which are more salient from the very beginning and thus have a lower change potential (Hypothesis 1). Two studies with elderly rehabilitation patients and middle-aged Nordic walkers empirically confirmed this hypothesis. For optimal exercisers (Study 1) and Nordic-walking maintainers (Study 2), the "fun" incentive (as a representative of activity-related incentive) increased more strongly than the "health" incentive (representative of purpose-related incentive) between the initiation and the maintenance phase of physical activity. Using a broader incentive measure, Study 2 revealed that the activity-related incentives of drop-outs decreased. Unexpectedly, the increase of activity-related incentives in maintainers was not significant.

In line with the principles of operant conditioning, the rewarding power of activity- and purpose-related incentives prompted us to hypothesize that activity-related incentives such as "fun" would be stronger predictors of physical activity than purpose-related incentives such as "health" (Hypothesis 2). According to the regularities of operant conditioning, activity-related incentives are more closely temporally related to the 
activity than purpose-related incentives and were thus assumed to have a stronger rewarding effect on the maintenance of behavior. In line with this assumption, the change in activity-related incentives predicted optimal exercising versus suboptimal exercising in Study 1 as well as maintenance versus drop-out in Study 2, whereas change in purpose-related incentives failed to account for a significant amount of maintenance variance. It is noteworthy that we found a consistent pattern of results for both hypotheses using different samples and partially different measures of incentives.

Our results provide initial evidence supporting our theoretical considerations and contributing to the fields of intrinsic motivation research and classical motivation research. Using the concept of activity- and purpose-related incentives (Rheinberg, 2008), and in doing so focusing on temporal distance from the activity as the most important differentiating characteristic of incentives, the terms "intrinsic" and "extrinsic" are given a different focus than has been applied in previous intrinsic motivation research (Deci \& Ryan, 1985). With the help of this focus, we were able to enhance the predictability of physical activity maintenance.

However, because our research focus was on the examination of activity- and purpose-related incentives, we did not consider further variables that might be important variables for sport and exercise adherence such as (changes in) self-efficacy or intention. Another limitation are our study samples. We tested very different samples in order to enhance the confidence in the generalizability of data. However, the majority of rehabilitation patients in the first study were male, whereas the majority of Nordic walkers in Study 2 were female and therefore it cannot be empirically excluded that gender and type of sport are confounded. Future studies are needed which replicate the study results for men and women, different age groups (e.g., children, adolescents) and different types of sports (e.g., competitive versus recreational sports) and address the limitations of the present studies.

The results of the present studies allow important conclusions for practical interventions to be drawn. For example, according to our results, health campaigns that aim to motivate individuals to adopt physical activity by generating activity-related incentives, such as, for example, having fun or feeling great while being physically active, will be less effective. For many people, working out is not fun in the beginning and exercising often does not feel good. Instead, fun and other positive qualities of exercising typically emerge in later phases when the activity has been performed for a certain amount of time. Promising fun from the very onset may frustrate individuals and facilitate dropout behavior because of unsatisfied expectancies (Rothman, 2000). Beginners rather need a realistic preview of the physical activity experience and self-regulatory strategies, such as action planning (Sniehotta, Scholz, \& Schwarzer, 2005) and other action control strategies (Kuhl \& Beckmann, 1985) such as vividly visualizing the positive consequences of physical activity in order to overcome the critical phase of being an beginner of physical activity. Once individuals have started to exercise, they require good conditions (e.g., challenge-skill balance; positive feedback) in order to discover activity-related incentives such as "fun" while performing the physical activity.

\section{REFERENCES}

Anderson, E. S., Wojcik, J. R., Winett, R. A., \& Williams, D. M.
(2006). Social-cognitive determinants of physical activity: The influence of social support, self-efficacy, outcome expectations, and self-regulation among participants in a church-based health promotion study. Health Psychology, 25, 510-520.

doi:10.1037/0278-6133.25.4.510

Atkinson, J. W. (1957). Motivational determinants of risk-taking behaviour. Psychological Review, 64, 359-372. doi: $10.1037 / \mathrm{h} 0043445$

Beckman, J., \& Heckhausen, H. (2008). Motivation as a function of expectancy and incentives. In J. Heckhausen, \& H. Heckhausen (Eds.), Motivation and action (pp. 99-136). Cambridge, UK: Cambridge University Press.

Blair, S. N., \& Connelly, J. C. (1996). How much exercise should we do? The case for moderate amounts and intensities of exercise. $R e$ search Quarterly for Exercise \& Sport, 67, 193-205.

Csikszentmihalyi, M. (1990). Flow: The psychology of optimal experience. New York: Harper \& Row.

Csikszentmihalyi, M., \& Rathunde, K. (1992). The measurement of flow in everyday live: Toward a theory of emergent motivation. In J. E. Jacobs (Ed.), Nebraska symposium on motivation (pp. 57-97). Lincoln: University of Nebraska Press.

Deci, E. L. (1971). Effects of externally mediated rewards on intrinsic motivation. Journal of Personality and Social Psychology, 18, 105115. doi:10.1037/h0030644

Deci, E. L., \& Ryan, R. M. (1985). Intrinsic motivation and self-determination in human behavior. New York: Plenum.

Deci, E. L., \& Ryan, R. M. (2000). The "what" and "why" of goal pursuits: Human needs and the self-determination of behavior. Psychological Inquiry, 11, 227-268. doi:10.1207/S15327965PLI1104 01

Dishman, R. K. (1994). Advances in exercise adherence. Champaign: Human Kinetics.

Duda, J. L. (1989). Goal perspectives and behavior in sport and exercise settings. In M. L. Maehr, \& C. Ames (Eds.), Advances in motivation and achievement: Motivation enhancing environments. Greenwich, CT: JAI Press.

Duda, J. L., \& Tappe, M. K. (1989). The personal incentives for exercise questionnaire: Preliminary development. Perceptual and Motor Skills, 68, 1122. doi:10.2466/pms.1989.68.3c.1122

Heckhausen, J., \& Heckhausen, H. (2008). Motivation and action. Cambridge, UK: Cambridge University Press. doi:10.1017/CBO9780511499821

Heckhausen, H., \& Rheinberg, F. (1980). Lernmotivation im unterricht, erneut betrachtet [Learning motivation in the classroom reconsidered]. Unterrichtswissenschaft, 8, 7-47.

Kuhl, J., \& Beckmann, J. (1985). Action control: From cognition to behavior. Heidelberg, Berlin, New York: Springer.

Liberman, N., \& Trope, Y. (1998). The role of feasibility and desirability considerations in near and distant future decisions: A test of temporal construal theory. Journal of Personality and Social Psychology, 75, 5-18. doi:10.1037/0022-3514.75.1.5

Markland, D., \& Hardy, L. (1993). The exercise motivations inventory: Preliminary development and validity of a measure of individuals' reasons for participation in regular physical exercise. Personality and Individual Differences, 15, 289-296. doi:10.1016/0191-8869(93)90219-S

Markland, D., \& Ingledew, D. K. (2007). Exercise participation motives. In M. S. Hagger, \& M. L. D. Chatzisarantis (Eds.), Intrinsic motivation and self-determination in exercise and sport. Human Kinetics.

McAuley, E., Wraith, S., \& Duncan, T. E. (1991). Self-efficacy, perceptions of success, and intrinsic motivation for exercise. Journal of Applied Social Psychology, 21, 139-155. doi:10.1111/j.1559-1816.1991.tb00493.x

McClelland, D. C. (1985). Human motivation. Glenview, IL: Scott, Foresman.

Pennington, G. L., \& Roese, N. J. (2003). Regulatory focus and temporal distance. Journal of Experimental Social Psychology, 39, 563-576. doi:10.1016/S0022-1031(03)00058-1

Prochaska, J. O., \& DiClemente, C. C. (1983). Stages and processes of 
self-change of smoking: Toward an integrative model of change. Journal of Consulting and Clinical Psychology, 51, 390-395. doi:10.1037/0022-006X.51.3.390

Prochaska, J. O., \& DiClemente, C. C. (1992). Toward a comprehensive model of change. In W. R. Miller, \& N. Heather (Eds.), Treating addictive behaviors: Process of change (pp. 3-27). New York: Plenum Press.

Prochaska, J. O., Velicer, W. F., Rossi, J. S., Goldstein, M. G., Marcus, B. H., Rakowski, W., Fiore, C., Harlow, L. L., Redding, C. A., Rosenbloom, D., \& Rossi, S. R. (1994). Stages of change and decisional balance for 12 problem behaviours. Health Psychology, 13, 39-46. doi:10.1037/0278-6133.13.1.39

Rheinberg, F. (2008). Intrinsic motivation and flow-experience. In H. Heckhausen, \& J. Heckhausen (Eds.), Motivation and action (pp. 323-348). Cambridge, UK: Cambridge University Press. doi:10.1017/CBO9780511499821.014

Rothman, A. J. (2000). Toward a theory-based analysis of behavioural maintenance. Health Psychology, 19, 64-69. doi:10.1037/0278-6133.19.Suppl1.64

Ryan, R. M., \& Deci, E. L. (2000). The darker and brighter sides of human existence: Basic psychological needs as a unifying concept. Psychological Inquiry, 11, 319-338. doi:10.1207/S15327965PLI1104 03

Sansone, C., \& Smith, J. L. (2000). Interest and self-regulation: The relation between having to and wanting to. In C. Sansone, \& J. M. Harackiewicz (Eds.), Intrinsic and extrinsic motivation (pp. 343-372). San Diego: Academic Press.

Scanlan, T. K., Carpenter, P. J., Schmidt, G. W., Simons, J. P., \& Keeler, B. (1993). An introduction of the sport commitment model. Journal of Sport \& Exercise Psychology, 15, 1-15.

Scheier, M. F., \& Carver, C. S. (1987). Dispositional optimism and physical well-being: The influence of generalized outcome expectancies on health. Journal of Personality, 55, 169-210. doi:10.1111/j.1467-6494.1987.tb00434.x

Schwarzer, R. (1992). Self-efficacy in the adoption and maintenance of health behaviours: Theoretical approaches and a new model. In R. Schwarzer (Ed.), Self-efficacy: Thought control of action (pp. 217-243). Washington DC: Hemisphere.

Shah, J. Y., \& Kruglanski, A. W. (2000). The structure and substance of intrinsic motivation. In C. Sansone, \& J. M. Harackiewicz (Eds.), Intrinsic and extrinsic motivation (pp. 105-127). San Diego: Academic Press.

Shanks, D. R., \& Dickinson, A. (1991). Instrumental judgement and performance under enhancement in action-outcome contingency and contiguity. Memory \& Cognition, 19, 353-360.

doi:10.3758/BF03197139

Skinner, B. F. (1938). The behaviour of organisms. New York: Appleton-Century-Crofts.

Sniehotta, F. F., Scholz, U., \& Schwarzer, R. (2005). Bridging the intention-behaviour gap: Planning, self-efficacy, and action control in the adoption and maintenance of physical exercise. Psychology \& Health, 20, 143-160. doi:10.1080/08870440512331317670

Tarpy, R. M., \& Sawabini, F. L. (1974). Reinforcement delay: A selective review of the last decades. Psychological Bulletin, 81, 984-997. doi:10.1037/h0037428

Trope, Y., \& Liberman, N. (2003). Temporal construal. Psychological Review, 110, 403-421. doi:10.1037/0033-295X.110.3.403

Vroom, V. H. (1964). Work and motivation. New York: Wiley.

Williams, D. M., Anderson, E. S., \& Winett, R. A. (2005). Review of outcome expectancies in physical activity research. Annals of Behavioral Medicine, 29, 70-79. doi:10.1207/s15324796abm2901_10

Wing, R. R. (2000). Cross-cutting themes in maintenance of behaviour change. Health Psychology, 19, 84-88. doi:10.1037/0278-6133.19.Suppl1.84 